TU-1034, UTHEP-694, KEK-TH-1940

\title{
Contravariant Gravity on Poisson Manifolds and Einstein Gravity
}

\author{
Yukio Kaneko ${ }^{\sharp 1}$, Hisayoshi Muraki ${ }^{\mathrm{b} 2}$ and Satoshi Watamura ${ }^{\sharp 3}$ \\ \# Tohoku University, \\ Graduate School of Science, \\ Aoba-ku, Sendai 980-8578, Japan \\ b University of Tsukuba, \\ Graduate School of Pure and Applied Sciences, \\ Tsukuba, Ibaraki 305-8571, Japan \\ † High Energy Accelerator Research Organization, \\ KEK Theory Center, \\ Tsukuba, Ibaraki 305-0801, Japan
}

\begin{abstract}
A relation between gravity on Poisson manifolds proposed in [1] and Einstein gravity is investigated. The compatibility of the Poisson and Riemann structures defines a unique connection, the contravariant Levi-Civita connection, and leads to the idea of the contravariant gravity. The Einstein-Hilbert-type action yields an equation of motion which is written in terms of the analog of the Einstein tensor, and it includes couplings between the metric and the Poisson tensor. The study of the Weyl transformation reveals properties of those interactions. It is argued that this theory can have an equivalent description as a system of Einstein gravity coupled to matter. As an example, it is shown that the contravariant gravity on a two-dimensional Poisson manifold can be described by a real scalar field coupled to the metric in a specific manner.
\end{abstract}

\footnotetext{
${ }^{1}$ e-mail: y_kaneko@tuhep.phys.tohoku.ac.jp

${ }^{2}$ e-mail: hmuraki@het.ph.tsukuba.ac.jp

3 e-mail: watamura@tuhep.phys.tohoku.ac.jp
} 


\section{Contents}

1 Introduction $\quad 1$

2 Overview of Riemannian Geometry on Poisson Manifolds 3

2.1 Contravariant Derivatives, Torsion and Curvature . . . . . . . . . . . . 3

2.2 Transformation Laws of $G^{i j}$ and $G=\operatorname{det} G^{i j} \ldots \ldots \ldots \ldots$

2.3 Divergence Theorem and Invariant Measure . . . . . . . . . . . . . . . 5

$\begin{array}{lll}3 & \text { Gravity on Poisson Manifolds } & 7\end{array}$

3.1 Contravariant Gravity Theory . . . . . . . . . . . . . . . . . . . 7

3.2 Weyl Transformation . . . . . . . . . . . . . . . . . . . . 9 9

4 Gravity on Two-Dimensional Poisson Manifolds $\quad 10$

5 Conclusion and Discussion 12

A Note on Riemannian Geometry on Poisson Manifolds $\quad 14$

B Relation between (Contravariant) Levi-Civita Connections 16

C Computations on Two-dimensional Manifolds 16

C.1 Calculation of Ricci Tensor _. . . . . . . . . . . . . . . . . . . . 17

C.2 Calculation of Weyl Transformation . . . . . . . . . . . . . . . 18

\section{Introduction}

Both Riemann and Poisson structures play significant roles in physics and mathematics. The former provides us with a geometrical intuition about spacetime and gravity, while the latter provides us with a geometrical intuition about the time evolution of a system and quantization. Here, we investigate the interplay between these two structures through a consideration on gravity on Poisson manifolds.

In quantum mechanics, it is well-known that a point in phase space can only be determined to an accuracy of the order of Planck's constant $\hbar$. The Heisenberg uncertainty principle can be regarded as a measure of the noncommutativity in the quantum world. The Poisson structure in phase space appears as a semi-classical approximation of the quantized system. Analogously, a Poisson structure of spacetime can be interpreted as a semi-classical approximation of the spatial noncommutativity. The idea of noncommutative spacetime can be traced back to Snyder [2]. Since then the application of this idea to physics, especially to gravity, has been discussed intensively in the literature $[3,4]$. For a review see for example $[5,6]$.

Recently, in the string theory context the concept of a minimal length is discussed. It is argued that it can be understood as an origin of noncommutativity in probing spacetime geometry $[7,8]$. For review see for example [9]. There is also a proposal of gravity on noncommutative 
spacetime in relation with deformation quantization [10,11]. It is based on the quantization of the diffeomorphism by twisting the Hopf algebra structure and there, a noncommutative algebra is formulated by the star product. See also [12].

One possible scenario would be that a gravity theory on noncommutative spaces gives an effective theory of quantum gravity. The noncommutativity should be very small and the effective theory can be described well by a semi-classical approximation. We can imagine that the relic of noncommutativity appears as a Poisson structure, and that the resulting Poisson structure should be compatible with the Riemann structure. From this point of view, gravity on noncommutative spaces and its semi-classical approximation, i.e. a gravity on Poisson manifolds, should be issues worthy of being studied.

The gravity on Poisson manifolds, which we refer here to as contravariant gravity, is formulated by Riemannian geometry compatible with a Poisson structure. The corresponding geometry is specified by a unique connection consisting of a Riemannian metric and a Poisson tensor, called the contravariant Levi-Civita connection. Such a geometry has been advocated in physics $[1,13]$ as well as in mathematics [14-18]. This geometry enables us to introduce the Poisson tensor as an intrinsic geometrical degree of freedom rather than a matter degree of freedom.

It is an interesting question to ask how the effect of the Poisson tensor incorporated in the geometry of contravariant gravity looks like. Can the effect be represented as a matter field in the usual Einstein gravity? Similar considerations are also found, for example, in Kaluza-Klein theory, where a five-dimensional metric governed by the Einstein-Hilbert action is decomposed into a four-dimensional metric, a $U(1)$ gauge field and a real scalar field, forming the fourdimensional Einstein-Maxwell dilaton theory. The aim of this article is to shed some light on these issues.

The organization of this article is as follows. In section 2, we give a brief review on the Riemannian geometry relevant for the contravariant gravity. The invariant measure and the divergence theorem are also discussed. In section 3 we give an action which describes the Einstein-Hilbert action in such a geometry. This theory, i.e. the contravariant gravity, incorporates the Poisson tensor as a geometrical degree of freedom on an equal footing with the metric, rather than a matter degree of freedom. Then, we discuss the equation of motion and a field redefinition given by a Weyl transformation. In section 4 we give a concrete example by applying the field redefinition to the action of our theory to the case of a two-dimensional Poisson-Riemannian manifold. It is shown that the theory has another description by Einstein gravity coupling to a real scalar field in a specific manner. The final section is devoted to summary and discussions. Appendices are for reference to the reader, making a brief introduction to the Lie algebroid induced by a Poisson structure and the Riemannian geometry based on the algebroid, and presenting some computational details. 


\section{Overview of Riemannian Geometry on Poisson Manifolds}

In this section we introduce a geometry describing gravity on a Poisson manifolds, which we call "contravariant gravity". This geometry is based on a unique connection specified by the metric-compatibility and the torsion-free conditions [1,13]. See also [14-18]. We give a summary on the definitions of geometrical notions as well as our notations in appendix A.

\subsection{Contravariant Derivatives, Torsion and Curvature}

Let $M$ be an $n$-dimensional Poisson-Riemannian manifold equipped with a Poisson tensor $\theta \in \Gamma\left(\wedge^{2} T M\right)$ and a Riemannian metric $G \in \Gamma\left(\otimes_{\text {Sym }}^{2}(T M)\right)$. The contravariant Levi-Civita connection $\bar{\nabla}: \Gamma\left(T^{*} M\right) \times \Gamma\left(T^{*} M\right) \rightarrow \Gamma\left(T^{*} M\right)$ is defined by

$$
\bar{\nabla}_{d x^{i}} d x^{j}=\bar{\Gamma}_{k}^{i j} d x^{k}
$$

where

$$
\bar{\Gamma}_{k}^{i j}=\frac{1}{2} G_{m k}\left(\theta^{i l} \partial_{l} G^{j m}+\theta^{j l} \partial_{l} G^{i m}-\theta^{m l} \partial_{l} G^{i j}+G^{l j} \partial_{l} \theta^{m i}+G^{l i} \partial_{l} \theta^{m j}+G^{l m} \partial_{l} \theta^{i j}\right),
$$

for $\theta^{i j}=\theta\left(d x^{i}, d x^{j}\right)$ and $G^{i j}=G\left(d x^{i}, d x^{j}\right)$ on a local patch $\left\{x^{i}\right\}$. For any one-forms $\xi=\xi_{i} d x^{i}$ and $\eta=\eta_{i} d x^{i}$, the definition of the connection (A.3) gives the following expression

$$
\bar{\nabla}_{\xi} \eta=\xi_{i}\left(\theta^{i j} \partial_{j} \eta_{k}+\bar{\Gamma}_{k}^{i j} \eta_{j}\right) d x^{k}
$$

In general, the contravariant derivative $\bar{\nabla}_{\xi}$ for an $(r, s)$-tensor field is given by

$$
\bar{\nabla}_{\xi} T_{j_{1} \cdots j_{s}}^{i_{1} \cdots i_{r}}=\xi_{k}\left(\theta^{k l} \partial_{l} T_{j_{1} \cdots j_{s}}^{i_{1} \cdots i_{r}}+\sum_{q=1}^{s} \bar{\Gamma}_{j_{q}}^{k l} T_{j_{1} \cdots i_{r} \cdots j_{s}}^{i_{1} \cdots i_{r}}-\sum_{p=1}^{r} \bar{\Gamma}_{l}^{k i_{p}} T_{j_{1} \cdots j_{s}}^{i_{1} \cdots i_{r}}\right)
$$

The contravariant Levi-Civita connection (2.2) is specified as the unique solution compatible with the metricity $\bar{\nabla}_{d x^{k}} G^{i j}=0$ and the torsion-free condition:

$$
\bar{T}(\xi, \eta):=\bar{\nabla}_{\xi} \eta-\bar{\nabla}_{\eta} \xi-[\xi, \eta]_{\theta}=0 .
$$

It also satisfies

$$
\overline{\mathcal{L}}_{\xi} G(\eta, \zeta)=G\left(\bar{\nabla}_{\xi} \eta, \zeta\right)+G\left(\eta, \bar{\nabla}_{\xi} \zeta\right)
$$

Here $\overline{\mathcal{L}}$ stands for the contravariant Lie derivative, acting on a function $f$ as

$$
\overline{\mathcal{L}}_{d x^{i}} f=\theta^{i j} \partial_{j} f
$$

and $[,]_{\theta}$ denotes the Koszul bracket, which is defined for one-forms $\xi=\xi_{i} d x^{i}$ and $\eta=\eta_{i} d x^{i}$ as

$$
[\xi, \eta]_{\theta}=\left(\xi_{k} \theta^{k l} \partial_{l} \eta_{i}-\left(\theta^{l k} \partial_{k} \xi_{i}+\partial_{i} \theta^{l k} \xi_{k}\right) \eta_{l}\right) d x^{i}\left(\equiv \overline{\mathcal{L}}_{\xi} \eta\right) .
$$


The contravariant Lie derivative acting on a tensor of type $(r, s)$ is defined by

$$
\overline{\mathcal{L}}_{\xi} T_{j_{1} \cdots j_{s}}^{i_{1} \cdots i_{r}}=\xi_{k} \theta^{k l} \partial_{l} T_{j_{1} \cdots j_{s}}^{i_{1} \cdots i_{r}}-\sum_{q=1}^{s}\left(M_{\xi}\right)^{l}{ }_{j_{q}} T_{j_{1} \cdots l \cdots j_{s}}^{i_{1} \cdots i_{r}}+\sum_{p=1}^{r}\left(M_{\xi}\right)^{i_{p}} T_{j_{1} \cdots j_{s}}^{i_{1} \cdots i_{r}}
$$

where we have introduced a matrix

$$
\left(M_{\xi}\right)^{i}{ }_{j}=\theta^{i k} \partial_{k} \xi_{j}+\partial_{j} \theta^{i k} \xi_{k} .
$$

For a derivation of these formulas, see reference [1].

The curvature tensor is defined by

$$
\begin{aligned}
\bar{R}_{l}^{k i j} d x^{l} & :=\left(\bar{\nabla}_{d x^{i}} \bar{\nabla}_{d x^{j}}-\bar{\nabla}_{d x^{j}} \bar{\nabla}_{d x^{i}}-\bar{\nabla}_{\left[d x^{i}, d x^{j}\right]_{\theta}}\right) d x^{k} \\
& =\left(\theta^{i m} \partial_{m} \bar{\Gamma}_{l}^{j k}-\theta^{j m} \partial_{m} \bar{\Gamma}_{l}^{i k}-\partial_{n} \theta^{i j} \bar{\Gamma}_{l}^{n k}+\bar{\Gamma}_{m}^{j k} \bar{\Gamma}_{l}^{i m}-\bar{\Gamma}_{m}^{i k} \bar{\Gamma}_{l}^{j m}\right) d x^{l}
\end{aligned}
$$

The corresponding Ricci tensor is defined by contracting an upper index of the curvature tensor with a lower one, $\bar{R}^{k j}:=\bar{R}_{l}^{k l j}$. Then, the scalar curvature is obtained by taking a contraction between the metric $G$ and the Ricci tensor:

$$
\bar{R}:=G_{i j} \bar{R}^{i j}=G^{i j} \bar{R}_{i j},
$$

where $G_{i k} G^{k j}=\delta_{i}^{j}$ and $\bar{R}_{i j}=G_{i k} G_{j l} \bar{R}^{k l}$. The curvature satisfies the following Bianchi identities

$$
\begin{aligned}
& \bar{R}_{l}^{k i j}+\bar{R}_{l}^{i j k}+\bar{R}_{l}^{j k i}=0, \\
& \bar{\nabla}_{d x^{k}} \bar{R}_{l}^{m i j}+\bar{\nabla}_{d x^{i}} \bar{R}_{l}^{m j k}+\bar{\nabla}_{d x^{j}} \bar{R}_{l}^{m k i}=0 .
\end{aligned}
$$

In addition to these identities, there is an additional identity

$$
\bar{R}^{m k i j}=-\bar{R}^{k m i j} .
$$

This property, together with the Bianchi identity, guarantees that the Ricci tensor is a symmetric tensor. We can also define an analog of the Einstein tensor of the form

$$
\overline{\mathcal{G}}^{i j}=\bar{R}^{i j}-\frac{1}{2} G^{i j} \bar{R},
$$

which satisfies

$$
\bar{\nabla}_{d x^{k}} G_{k i} \overline{\mathcal{G}}^{i j}=0
$$

owing to the Bianchi identities (2.14) and (2.15).

\subsection{Transformation Laws of $G^{i j}$ and $G=\operatorname{det} G^{i j}$}

The contravariant Lie derivative (2.9) of the metric tensor $G^{i j}$ can be written in terms of the contravariant derivatives (2.4) as

$$
\overline{\mathcal{L}}_{\xi} G^{k l}=\bar{\nabla}_{d x^{k}}\left(\xi_{i} G^{i l}\right)+\bar{\nabla}_{d x^{l}}\left(\xi_{i} G^{k i}\right),
$$


where we used a fact that $\bar{\nabla}_{d x^{k}} G^{i j}=0$. This is a contravariant counterpart of $\mathcal{L}_{X} g_{i j}=\nabla_{i} X_{j}+$ $\nabla_{j} X_{i}$ in the usual Riemannian geometry. It is also shown that the Kronecker delta is invariant under the contravariant Lie derivatives: $\overline{\mathcal{L}}_{\xi} \delta_{l}^{k}=0$. Then we obtain

$$
\overline{\mathcal{L}}_{\xi} G_{i j}=-G_{i k}\left(\overline{\mathcal{L}}_{\xi} G^{k l}\right) G_{l j}
$$

Introducing $G^{-1}=\left(\operatorname{det} G^{i j}\right)^{-1}=\operatorname{det} G_{i j}$, we find

$$
\overline{\mathcal{L}}_{\xi} \sqrt{G^{-1}}=\frac{1}{2} \sqrt{G^{-1}} G^{i j} \overline{\mathcal{L}}_{\xi} G_{i j}=-\sqrt{G^{-1}} \bar{\nabla}_{d x^{i}} \xi_{i} .
$$

Using an identity

$$
\sqrt{G^{-1}} \bar{\Gamma}_{j}^{j i}=-\partial_{j}\left(\sqrt{G^{-1}} \theta^{i j}\right)
$$

we find a useful relation:

$$
\sqrt{G^{-1}} \bar{\nabla}_{d x^{i}} \xi_{i}=\partial_{j}\left(\sqrt{G^{-1}} \theta^{i j} \xi_{i}\right)+2 \sqrt{G^{-1}} \bar{\Gamma}_{j}^{j i} \xi_{i}
$$

There is a crucial difference between the above relation and the one in usual Riemannian geometry: The covariant counterpart is given by

$$
\sqrt{g} \nabla_{i} X^{i}=\partial_{i}\left(\sqrt{g} X^{i}\right)
$$

which plays an important role in the proof of the general covariance of the action integral. On the other hand, in the contravariant geometry the quantity $\bar{\Gamma}_{j}^{j i}$ in (2.23) arises as an obstruction to the "covariance" of the naïve integral measure $\sqrt{G^{-1}} d^{n} x$. This obstruction forces us to introduce an additional factor into the invariant measure. For later purpose, we note the following relation: For any scalar $F$, we have

$$
\overline{\mathcal{L}}_{\xi}\left(F \sqrt{G^{-1}}\right)=\partial_{j}\left(F \sqrt{G^{-1}} \xi_{i} \theta^{i j}\right)+F \sqrt{G^{-1}} \theta^{i j}\left(\partial_{i} \xi_{j}-\partial_{j} \xi_{i}\right),
$$

which is shown by using (2.21) and (2.22).

\subsection{Divergence Theorem and Invariant Measure}

As discussed above, a naïve integral measure $\sqrt{G^{-1}}$ fails to give a measure invariant under the contravariant Lie derivatives. The invariance is broken by the existence of $\bar{\Gamma}_{j}^{j i}$ in (2.23). The removal of this obstruction has already been discussed in [1], where an invariant measure is constructed by multiplying $\sqrt{G^{-1}}$ by a scalar factor $e^{\phi}$. However, we conclude that it is not possible to obtain an integral which is fully invariant under the contravariant Lie derivatives, due to (2.25). We give details on this issue in the latter half of this subsection. 
Divergence Theorem As in usual Riemannian geometry, we can also formulate the divergence theorem (2.24) in contravariant differential calculus. An invariant measure $e^{\phi} \sqrt{G^{-1}}$ satisfies the "divergence theorem" if it holds

$$
e^{\phi} \sqrt{G^{-1}} \bar{\nabla}_{d x^{i}} \xi_{i}=\partial_{j}\left(e^{\phi} \sqrt{G^{-1}} \xi_{i} \theta^{i j}\right) .
$$

This requirement imposes a condition on $\phi$ :

$$
\theta^{i j} \partial_{j} \phi=2 \bar{\Gamma}_{j}^{j i}=-\frac{2}{\sqrt{G^{-1}}} \partial_{j}\left(\sqrt{G^{-1}} \theta^{i j}\right) .
$$

The proof is as follows: Take the derivation in right-hand side of (2.26) then substitute (2.27) for $\theta^{i j} \partial_{j} \phi$. Using the formula of $\bar{\Gamma}_{j}^{j i}$, we obtain the left-hand side.

Note that $\bar{\Gamma}=\bar{\Gamma}_{i}^{i j} \partial_{j}$ defines a vector field (see appendix A). By a straightforward computation, this vector $\bar{\Gamma}$ turns out to be $d_{\theta}$-closed. Thus, it makes sense to ask whether the vector $\bar{\Gamma}$ is $d_{\theta}$-exact or not. The condition $(2.27)$ is nothing but $d_{\theta}$-exactness of $\bar{\Gamma}$. In this article, we assume that the vector field $\bar{\Gamma}$ is a trivial element of the first $d_{\theta}$-cohomology, i.e., there exists a function $\phi$ satisfying the equation (2.27).

In the case where the Poisson tensor is invertible, i.e. there exists a symplectic structure, the equation (2.27) can be solved by the ansatz

$$
\phi=-\log \left(\operatorname{det}\left(\theta^{i j}\right) \operatorname{det}\left(G_{k l}\right)\right)
$$

It is straightforward to show that this ansatz solves the condition (2.27).

Invariant Measure Here we discuss about the measure which is invariant under the transformation generated by the contravariant Lie derivatives. We see that it is impossible to obtain an integral which has full invariance. It turns out that we have to restrict the transformation by imposing a condition on the transformation parameter.

Let us first consider the general condition for the invariant measure:

$$
\overline{\mathcal{L}}_{\xi}\left(e^{\phi} \sqrt{G^{-1}}\right)=C \partial_{j}\left(e^{\phi} \sqrt{G^{-1}} \xi_{i} \theta^{i j}\right) .
$$

The above requirement guarantees the invariance of the measure $e^{\phi} \sqrt{G^{-1}}$ itself ${ }^{4}$. In order to define an invariant action, we have also to require invariance of an integration with a scalar function. The condition (2.29) gives the relation for any scalar integrand $F$

$$
\overline{\mathcal{L}}_{\xi}\left(e^{\phi} \sqrt{G^{-1}} F\right)=\partial_{j}\left(e^{\phi} \sqrt{G^{-1}} \xi_{i} \theta^{i j} F\right)+(C-1) \partial_{j}\left(e^{\phi} \sqrt{G^{-1}} \xi_{i} \theta^{i j}\right) F .
$$

Hence, we have to require $C=1^{5}$.

\footnotetext{
${ }^{4} \operatorname{In}[1] C=-1$ is realized for any one-form $\xi$, by setting $\theta^{i j} \partial_{j} \phi=\bar{\Gamma}_{j}^{j i}$. This condition for $\phi$ can be re-expressed as $\partial_{j}\left(e^{\phi} \sqrt{G^{-1}} \theta^{i j}\right)=0$.

${ }^{5}$ One may impose $\partial_{j}\left(e^{\phi} \sqrt{G^{-1}} \xi_{i} \theta^{i j}\right)=0$. But this condition involves a one-form $\xi$ and the argument is carried out in a parallel manner with that in the main body. This is reduced to the case when we set $\theta^{i j} \partial_{j} \phi=\bar{\Gamma}_{j}^{j i}$.
} 
However, the condition (2.29) with $C=1$ can not be realized for a general one-form $\xi$, since the second term in (2.25) can not be canceled out by any choice of $\phi$. Therefore, in order to satisfy (2.29), the second term on the right-hand side in equation (2.25) should vanish. This means that we have to restrict the transformation to the covariant Lie derivatives induced by the one-form $\xi$ satisfying

$$
\theta(d \xi)=\theta^{i j}\left(\partial_{i} \xi_{j}-\partial_{j} \xi_{i}\right)=0
$$

When the parameter is restricted by this condition, from (2.25), we automatically obtain (2.29) with $C=1$.

\section{Gravity on Poisson Manifolds}

In this section, we investigate a gravity theory based on the contravariant Levi-Civita connection. We investigate the analog of the Einstein-Hilbert action defined by the scalar curvature (2.13) [1]. First we derive the corresponding equation of motion and show that it is given by an analog of the Einstein tensor. Then, we perform the Weyl transformation for this Einstein-Hilbert action and discuss how the Poisson tensor couples to the gravity.

\subsection{Contravariant Gravity Theory}

As we discussed in the previous section, in order to define the invariant action with the scalar curvature, we restrict the transformation parameter 1-form $\xi$ as $\theta(d \xi)=0$. Furthermore, we take the measure satisfying the divergence theorem (2.26). Thus, the measure is defined with the factor $e^{\phi}$ defined by the condition (2.27).

The action of contravariant gravity is defined by

$$
S=\int d^{n} x e^{\phi} \sqrt{G^{-1}} \bar{R}
$$

where

$$
\theta^{i j} \partial_{j} \phi=-\frac{2}{\sqrt{G^{-1}}} \partial_{j}\left(\sqrt{G^{-1}} \theta^{i j}\right)
$$

We note that a variation of the scalar function $\phi=\phi[\theta, G]$ must also be taken into account when we take the variation of the metric to obtain the equation of motion.

Varying the Lagrangian density with respect to the metric, we find

$$
\delta\left(e^{\phi} \sqrt{G^{-1}} \bar{R}\right)=e^{\phi} \sqrt{G^{-1}} G_{i j} \delta \bar{R}^{i j}+e^{\phi} \sqrt{G^{-1}} \delta G_{i j} \bar{R}^{i j}+e^{\phi} \delta \sqrt{G^{-1}} \bar{R}+\delta \phi e^{\phi} \sqrt{G^{-1}} \bar{R} .
$$

Since the scalar $\phi$ is a solution of the partial differential equation (2.27), we must also consider its variation $\delta \phi$ :

$$
\delta \phi=\phi[\theta, G+\delta G]-\phi[\theta, G]
$$


This implies

$$
\begin{aligned}
\theta^{i j} \partial_{i} \delta \phi & =\theta^{i j} \partial_{i}(\phi[\theta, G+\delta G]-\phi[\theta, G]) \\
& =-2 \theta^{i j} \partial_{i} \delta\left(\log \sqrt{G^{-1}}\right) .
\end{aligned}
$$

We find that a solution is given by

$$
\delta \phi=-2 \delta\left(\log \sqrt{G^{-1}}\right)=\frac{-2}{\sqrt{G^{-1}}} \delta \sqrt{G^{-1}} .
$$

To derive the variation of the Ricci tensor, we first examine the variation of the Riemann tensor $\delta \bar{R}_{l}^{k i j}$. As mentioned in appendix A, the difference of the connection coefficients $\delta \bar{\Gamma}_{k}^{i j}=$ $\bar{\Gamma}_{k}^{i j}[G+\delta G, \theta]-\bar{\Gamma}_{k}^{i j}[G, \theta]$ is a tensor, and thus, its contravariant derivative is well-defined:

$$
\bar{\nabla}_{d x^{i}} \delta \bar{\Gamma}_{l}^{j k}=\theta^{i m} \partial_{m} \delta \bar{\Gamma}_{l}^{j k}+\delta \bar{\Gamma}_{m}^{j k} \bar{\Gamma}_{l}^{i m}-\delta \bar{\Gamma}_{l}^{m k} \bar{\Gamma}_{m}^{i j}-\delta \bar{\Gamma}_{l}^{j m} \bar{\Gamma}_{m}^{i k}
$$

We find

$$
\delta \bar{R}_{l}^{k i j}=\bar{\nabla}_{d x^{i}} \delta \bar{\Gamma}_{l}^{j k}-\bar{\nabla}_{d x^{j}} \delta \bar{\Gamma}_{l}^{i k}
$$

where we used $\bar{\Gamma}_{m}^{i j}-\bar{\Gamma}_{m}^{j i}=\partial_{m} \theta^{i j}$. Using the above relation, the variation of the Ricci tensor is obtained by taking a contraction with the metric. Using (2.23) and the definition of $\phi,(2.27)$, we obtain

$$
e^{\phi} \sqrt{G^{-1}} G_{i j} \delta \bar{R}^{i j}=\partial_{m}\left[e^{\phi} \theta^{l m} \sqrt{G^{-1}}\left(G_{i j} \delta \bar{\Gamma}_{l}^{j i}-G_{i l} \delta \bar{\Gamma}_{j}^{j i}\right)\right]
$$

where we used the divergence theorem (2.26). As a result, the variation of the action with respect to the metric reads

$$
\delta S=\int d^{n} x\left\{e^{\phi} \sqrt{G^{-1}}\left(\bar{R}^{i j}-\frac{1}{2} G^{i j} \bar{R}\right) \delta G_{i j}+\partial[\ldots]\right\} .
$$

Thus, the equation of motion of the metric $G_{i j}$ is

$$
\bar{R}^{i j}-\frac{1}{2} G^{i j} \bar{R}=0
$$

The left-hand side is nothing but the analog of the Einstein tensor $\overline{\mathcal{G}}^{i j}$ defined in (2.17).

Let us comment on the possible terms one may add to the Einstein-Hilbert action. As usual, one can add a cosmological constant term

$$
S_{c}=2 \Lambda \int d^{n} x e^{\phi} \sqrt{G^{-1}}
$$

Using the equation (3.6), we find that the variation of the cosmological constant term gives

$$
\begin{aligned}
\delta S_{c} & =2 \Lambda \int d^{n} x\left(\delta \phi e^{\phi} \sqrt{G^{-1}}+e^{\phi} \delta \sqrt{G^{-1}}\right) \\
& =-2 \Lambda \int d^{n} x e^{\phi} \delta \sqrt{G^{-1}}=-\Lambda \int d^{n} x e^{\phi} \sqrt{G^{-1}} G^{i j} \delta G_{i j} .
\end{aligned}
$$


It contributes to the equation of motion as follows

$$
\bar{R}^{i j}-\frac{1}{2} G^{i j} \bar{R}=\Lambda G^{i j}
$$

In principle, we can add any diffeomorphism invariant terms to the Einstein-Hilbert-type action, such as $\bar{R}^{2}$, the Weyl tensor etc. It is also interesting to discuss an analog of the Gibbons-Hawking term when a Poisson manifold has boundaries. In the following section, we mainly focus on the action of the Einstein-Hilbert term (3.1).

\subsection{Weyl Transformation}

We have introduced a scalar degree of freedom $\phi$ to keep the divergence theorem (2.26), and derived the Einstein equation (3.11). However, its physical interpretation is less clear. Naïvely, one might think that it originates from a dilaton field. In this subsection, we consider the Weyl transformation and provide transformation rules for the Riemann tensor and the scalar field $\phi$. We also show that an analog of the Weyl tensor can be defined.

Let us consider the Weyl transformation given by

$$
G^{i j} \longrightarrow \tilde{G}^{i j}=e^{2 \Omega} G^{i j}
$$

where $\Omega$ is an arbitrary function on the manifold. We assume that the Poisson tensor does not change under the Weyl transformation. The coefficients $\bar{\Gamma}_{k}^{i j}$ of the contravariant Levi-Civita connection are transformed as

$$
\tilde{\bar{\Gamma}}_{k}^{i j}=\bar{\Gamma}_{k}^{i j}+G_{m k}\left(G^{j m} \theta^{i l}+G^{i m} \theta^{j l}-G^{i j} \theta^{m l}\right) \partial_{l} \Omega .
$$

The curvature tensor becomes

$$
\tilde{\bar{R}}_{l}^{k i j}=\bar{R}_{l}^{k i j}+\bar{L}_{l}^{k i j}
$$

where

$$
\begin{aligned}
& \bar{L}_{l}^{k i j}=\delta_{l}^{j} B^{i k}-\delta_{l}^{i} B^{j k}-G^{j k} G_{l m} B^{i m}+G^{i k} G_{l m} B^{j m}, \\
& B^{i k}=\bar{\nabla}_{d x^{i}}\left(\theta^{k j} \partial_{j} \Omega\right)-\left(\theta^{i j} \partial_{j} \Omega\right)\left(\theta^{k l} \partial_{l} \Omega\right)+\frac{1}{2} G^{i k} G_{j l}\left(\theta^{j m} \partial_{m} \Omega\right)\left(\theta^{l n} \partial_{n} \Omega\right) .
\end{aligned}
$$

For $n$-dimensional manifolds, the Weyl transformations of the Ricci tensor and the corresponding Ricci scalar are

$$
\begin{aligned}
& \tilde{\bar{R}}^{i j}=\bar{R}^{i j}-(n-2) B^{j i}-G^{i j} G_{k l} B^{k l}, \\
& e^{2 \Omega} \tilde{\bar{R}}=\bar{R}-2(n-1) G_{i j} B^{i j} .
\end{aligned}
$$

Here, we consider the Weyl transformation of the Einstein-Hilbert action. We note that under the Weyl transformation the scalar field $\phi$ transforms

$$
\phi \rightarrow \tilde{\phi}=\phi+2 n \Omega,
$$


since the scalar field $\phi$ solves the partial differential equation (2.27). It yields

$$
e^{\tilde{\phi}} \sqrt{\tilde{G}^{-1}} \tilde{\bar{R}}=e^{(n-2) \Omega} e^{\phi} \sqrt{G^{-1}}\left(\bar{R}+G_{k j} \bar{L}_{i}^{k i j}\right) .
$$

Therefore, we can eliminate the scalar field $\phi$, which appears in the prefactor of the EinsteinHilbert action, by performing the Weyl transformation. We will show that the two-dimensional contravariant gravity theory can be rephrased in terms of the usual Einstein-Hilbert action.

It is notable that we also find an analog of the Weyl tensor of the form

$$
\begin{aligned}
C_{l}^{k i j}= & \bar{R}_{l}^{k i j}+\frac{1}{n-2}\left(\delta_{l}^{j} \bar{R}^{k i}-\delta_{l}^{i} \bar{R}^{k j}-G^{j k} G_{l m} \bar{R}^{m i}+G^{i k} G_{l m} \bar{R}^{m j}\right) \\
& +\frac{1}{(n-1)(n-2)}\left(\delta_{l}^{j} G^{i k}-\delta_{l}^{i} G^{j k}\right) \bar{R} .
\end{aligned}
$$

We can check the Weyl invariance of the Weyl tensor as follows. According to the equations (3.21) and (3.22), we find that

$$
B^{i j}=\frac{1}{n-2}\left(\bar{R}^{i j}-\tilde{\bar{R}}^{i j}\right)+\frac{1}{2(n-1)(n-2)}\left(G^{i j} \bar{R}-\tilde{G}^{i j} \tilde{\bar{R}}\right) .
$$

By substituting (3.26) into the equation (3.18), we see that the Weyl tensor $C_{l}^{k i j}$ is invariant under the Weyl transformation.

Before closing this section, we remark the local scale transformation of the Poisson tensor. In the contravariant gravity theory, we consider both the Poisson tensor and the metric tensor as geometric objects, and we should treat them equally and independently. As we have seen, we can consider any local parameter $\Omega$ for the Weyl transformation $\tilde{G}^{i j}=e^{2 \Omega} G^{i j}$. However, we must restrict the local parameter $\omega$ for the transformation $\hat{\theta}^{i j}=e^{2 \omega} \theta^{i j}$, because the Poisson condition does not hold unless $d_{\theta} \omega=0$. Therefore, compared to the Weyl transformation, the scale transformation of the Poisson tensor is limited.

\section{Gravity on Two-Dimensional Poisson Manifolds}

In the previous section, we analyzed general properties of the gravity theory on a PoissonRiemannian manifold. For a more concrete discussion, we consider the case of a two-dimensional Poisson-Riemannian manifold $\left(M, g=G^{-1}, \theta\right)$ in detail. We reveal the interaction terms between the metric and the Poisson tensor explicitly.

In two dimensions the Poisson tensor has a form

$$
\theta^{i j}=\left(\begin{array}{cc}
0 & \theta \\
-\theta & 0
\end{array}\right) .
$$

For any function $\theta$ on the manifold, the Poisson condition is satisfied because no three-vector exists on two-dimensional manifolds. Here, we mention that the combination

$$
\sqrt{G^{-1}} \theta=\sqrt{g} \theta
$$


is invariant under diffeomorphisms, since $\theta$ behaves in the same way as $d^{2} x$. It is convenient to use the combination (4.2) to discuss interactions between the Poisson tensor $\theta^{i j}$ and the metric $g_{i j}=\left(G^{-1}\right)_{i j}, g^{i j}=G^{i j}$.

Next, we consider the constraint on the scalar field $\phi$, which is given by (2.27). On the two-dimensional manifold $M$, the equation reduces to

$$
\sqrt{G^{-1}} \theta \partial_{i} \phi=-2 \partial_{i}\left(\sqrt{G^{-1}} \theta\right)
$$

The general solution for $\phi$ is given by

$$
e^{\phi}=C\left(\sqrt{G^{-1}} \theta\right)^{-2}
$$

where $C$ is an arbitrary constant. Therefore the Einstein-Hilbert-type action (3.1) takes the form

$$
S=C \int d^{2} x \frac{1}{\theta^{2} G^{-1}} \sqrt{G^{-1}} \bar{R}
$$

Field Redefinition We start with the action of the form

$$
S=\int d^{2} x \frac{1}{\theta^{2} G^{-1}} \sqrt{G^{-1}} \bar{R}
$$

where the constant $C$ in eq. (4.5) is set to unity for simplicity. The action can be written as (see appendix C.1)

$$
S=\int d^{2} x \sqrt{g}\left(R+\frac{2}{\sigma} \nabla^{i} \partial_{i} \sigma-\frac{1}{\sigma^{2}} \partial^{i} \sigma \partial_{i} \sigma\right)
$$

with introducing a scalar degree of freedom defined by

$$
\sigma=\sqrt{G^{-1}} \theta
$$

where the symbol $\nabla$ represents a covariant derivative with respect to the usual Levi-Civita connection. In this article, we assume that the Poisson manifold does not have a boundary. Then, we can do an integration by parts freely and obtain the action of the form

$$
S=\int d^{2} x \sqrt{g}\left(R+g^{i j} \partial_{i} \pi \partial_{j} \pi\right)
$$

where we have introduced a redefined scalar degree of freedom $\pi$ defined by

$$
\pi=\log \sigma=\log (\sqrt{g} \theta) .
$$

Therefore, we get a free scalar field theory on a two-dimensional Riemannian manifold.

On the other hand, we could have considered the field redefinition by performing a Weyl transform applying to (4.6). The observation here gives a non-trivial consistency check to the formula under the Weyl transformation. The resultant action is

$$
S=\int d^{2} x \frac{1}{\theta^{2} G^{-1}} \sqrt{G^{-1}}\left(\bar{R}+G_{k j} \bar{L}_{i}^{k i j}\right),
$$


with setting the Weyl transformation parameter as

$$
\Omega=\log \sigma
$$

We must reach the same result as above. Again, referring to appendix C.1, the action can be written as

$$
S=\int d^{2} x \sqrt{g}\left(R+\frac{2}{\sigma} \nabla^{i} \partial_{i} \sigma-\frac{1}{\sigma^{2}} \partial^{i} \sigma \partial_{i} \sigma+\frac{1}{\sigma^{2}} G_{k j} L_{i}^{k i j}\right)
$$

With some manipulations we find (see appendix C.2)

$$
G_{k j} L_{i}^{k i j}=2 \partial^{i} \sigma \partial_{i} \sigma-2 \sigma \nabla_{i} \partial^{i} \sigma
$$

and these terms turn out to give a surface term, together with the invariant measure:

$$
\int d^{2} x \frac{\sqrt{g}}{\sigma^{2}} G_{k j} L_{i}^{k i j}=-\int d^{2} x \partial_{i}\left(\sqrt{g} \frac{1}{\sigma} G^{i j} \partial_{j} \sigma\right),
$$

which does not change the equation of motion, but modifies the action equivalently to doing an integration by parts. Then, the contravariant Einstein-Hilbert action becomes

$$
\begin{aligned}
S & =\int d^{2} x \sqrt{g}\left(R+\frac{1}{\sigma^{2}} G^{i j} \partial_{i} \sigma \partial_{j} \sigma\right) \\
& =\int d^{2} x \sqrt{g}\left(R+g^{i j} \partial_{i} \pi \partial_{j} \pi\right),
\end{aligned}
$$

as obtained in the above.

Cosmological Constant Under the solution of a scalar field (4.4), the cosmological constant term (3.12) gives an interaction term of $\sigma$ :

$$
S_{c}=2 \Lambda \int d^{2} x e^{\phi} \sqrt{G^{-1}}=2 \Lambda \int d^{2} x \sqrt{g} \sigma^{-2}
$$

In terms of $\pi$, the total action is

$$
S+S_{c}=C \int d^{2} x \sqrt{g}\left(R+g^{i j} \partial_{i} \pi \partial_{j} \pi+2 \Lambda e^{-2 \pi}\right) .
$$

\section{Conclusion and Discussion}

In this paper, we studied the gravity theory based on the contravariant Levi-Civita connection on Poisson manifolds. In particular, we investigated the Einstein-Hilbert-type action given by the scalar curvature constructed from the contravariant Levi-Civita connection. We analyzed the property of the invariant measure and found that for the transformation generated by the contravariant Lie derivative $\overline{\mathcal{L}}_{\xi}$, the invariant measure can be defined only when we impose the condition $\theta(d \xi)=0$ on the parameter one-form $\xi$. 
We also discussed about the divergence theorem. We found that it is necessary to introduce an additional factor $e^{\phi}$ in the invariant measure which satisfies the partial differential equation (2.27). The reason is that as shown in (2.23), the divergence given by the contravariant LeviCivita connection does not become a surface term with the naïve invariant measure. In terms of the $d_{\theta}$-cohomology, the differential equation (2.27) which defines $\phi$ implies that the one-vector $\bar{\Gamma}_{i}^{i j} \partial_{j}$ is $d_{\theta}$-exact. Although the vector field $\bar{\Gamma}_{i}^{i j} \partial_{j}$ is $d_{\theta}$-closed for any Poisson tensor $\theta$, it is not necessarily exact in general. In this article we assume that $\bar{\Gamma}_{i}^{i j} \partial_{j}$ is exact. Therefore, the scalar field $\phi$ is always expressed by the metric and the Poisson tensor. It would be interesting to consider a generalization of the differential equation to the gravity theory with nontrivial $d_{\theta}$-cohomology.

We proposed an analog of the Einstein-Hilbert action (3.1) with an invariant measure which is consistent with the divergence theorem. Then, we derived the equation of motion for the metric $G^{i j}$. This equation is written by the analog of the Einstein tensor (2.17) in the contravariant gravity theory. Note that we can also add a cosmological term.

The Weyl transformation of the Riemann tensor in the contravariant theories is established. Its behavior under the Weyl transformation is very similar to the behavior of the ordinary Riemann tensor of the usual Levi-Civita connection. The scalar field $\phi$ transforms under the Weyl transformation is compatible with the condition (2.27). In addition, we could absorb the dilaton-like coupling of the scalar field $\phi$ in the contravariant Einstein-Hilbert action, i.e. we could move to the Einstein frame in the contravariant gravity theory.

Furthermore, we discussed in detail the interaction between the Poisson tensor and the metric on the two-dimensional Poisson manifold. In this case, we solved the differential equation (2.27) without any assumption for the Poisson tensor and the metric, and we gave an explicit form of the contravariant Einstein-Hilbert action. In addition, we showed that the action can be expressed by the ordinary Einstein-Hilbert action coupled to a free scalar field $\pi$. We also discussed the interaction term induced by the cosmological constant term. The interaction turned out to be described by an exponential potential of the scalar field $\pi$ in the usual Einstein theory.

There are some interesting future directions to study the contravariant gravity theory. In general, we can add all possible terms which are compatible with contravariant gravity, for instance a kinetic term of the Poisson tensor. It would also be natural to ask whether one can move on to noncommutative spaces, applying the quantization of the Poisson structure in [19]. In this sense, as we mentioned in the introduction, our theory may be related to gravity theories on noncommutative spaces discussed in the purely mathematical as well as in the string/Mtheoretical literature, such as [3,4,9-12,20-24].

Recently, a notion of emergent geometry has been discussed within matrix theoretic and noncommutative geometric frameworks [25-29]. In those considerations, the Poisson tensor plays a role as significant as the metric. Recalling that our theory handles the Poisson tensor on an equal footing with the metric, it would be interesting to apply our framework to geometries arising from matrix models of superstring theory and M-theory, especially those equipped with the Kähler structure [30-33]. 
It would be also interesting to use our framework for the description of effective theories of superstring theory. Actually, our original motivation to introduce the gravity with the (quasi-) Poisson structure and, as it turned out, with the contravariant Levi-Civita connection was to describe aspects of T-duality such as non-geometric background fields, e.g. R-flux [1,13,34-40]. In these references, the same connection is utilized to formulate the non-geometric fluxes. On the other hand, the non-geometric background with Poisson structure is also analyzed by using the supergeometric method in a recent study [41]. It is also interesting to discuss the gravity theory with Poisson structure from the supergeometric point of view [42-46].

Finally, one can consider the contravariant gravity theory in odd-dimensional spacetime where the Poisson tensor must be degenerate. Note that we did not need the non-degeneracy of the Poisson tensor in our analysis. The degeneracy would clarify the difference between the gravity theory based on the contravariant Levi-Civita connection and that of the usual LeviCivita connection, since the degeneracy of the Poisson tensor gives rise to a degeneracy of the contravariant Lie derivatives, which then might fail to generate full diffeomorphisms. This defect of diffeomorphisms would give new aspects in the gravity theory. The dimensional reduction has a similar effect. Along this line, a dimensional reduction has been already discussed in part on the Courant algebroid level [47]. As a related study, the (generalized) Einstein-Hilbert actions and their dimensional reductions have been also discussed from a viewpoint based on the Courant/Leibniz algebroid by $[48,49]$. Besides, in odd-dimensional spaces there are the contact structure, the Jacobi structure and the Nambu-Poisson structure, as specific structures related to the Poisson structure. It is an open question how the gravity theory coupled to such structures could be realized. The investigation of these structures is a near future project and we hope that this will shed some light on the properties of odd-dimensional noncommutative and nonassociative spaces.

\section{Acknowledgments}

The authors would like to give thanks to Tsuguhiko Asakawa for helpful comments and important contributions at the earlier stage of this work, Ursula Carow-Watamura for careful reading and improving our manuscript, Taiki Bessho, Marc Andre Heller, Noriaki Ikeda, Goro Ishiki, Branislav Jurčo, Shinpei Kobayashi, Takaki Matsumoto, Yuta Sekiguchi and Thomas Strobl for fruitful discussions. YK is supported by Tohoku University Division for Interdisciplinary Advanced Research and Education (DIARE). HM is supported in part by the Iwanami Fujukai Foundation.

\section{A Note on Riemannian Geometry on Poisson Manifolds}

In this section we give a brief note on how one can build the notion of Riemannian geometry on Poisson manifolds. First, in order to fix our conventions, we give a quick review on a Lie algebroid of one-forms induced by a Poisson structure on Poisson manifolds. Precise statements 
and detailed arguments can be found in $[1,13]$ and also in [14-18]. Based on the Lie algebroid, we define a contravariant affine connection, its torsion and curvature tensors.

Lie Algebroid on Poisson Manifolds Let $M$ be an $n$-dimensional Poisson manifold equipped with a Poisson tensor $\theta \in \Gamma\left(\wedge^{2} T M\right)$, which satisfies the condition $[\theta, \theta]_{S}=0$. Here $[\cdot, \cdot]_{S}$ stands for the Schouten-Nijenhuis bracket. A Lie algebroid on the manifold $M$ is defined by a triple $\left(T^{*} M, \theta,[\cdot, \cdot]_{\theta}\right): T^{*} M$ is the cotangent bundle over $M$; the anchor map $\theta: T^{*} M \rightarrow T M$ is given by the Poisson tensor through $\theta(\xi)=\bar{\iota}_{\xi} \theta$ for $\xi \in \Gamma\left(T^{*} M\right)$; and the Lie bracket is given by

$$
[\xi, \eta]_{\theta}=\mathcal{L}_{\theta(\xi)} \eta-i_{\theta(\eta)} d \xi
$$

called the Koszul bracket.

An exterior derivative $d_{\theta}=[\theta, \cdot]_{S}$, an interior product $\bar{\iota}_{\zeta}$ and a Lie derivative $\overline{\mathcal{L}}_{\zeta}=\left\{d_{\theta}, \bar{\iota}_{\zeta}\right\}$ with respect to a one-form $\zeta \in \Gamma\left(T^{*} M\right)$ acting on polyvectors $\Gamma\left(\wedge^{\bullet} T M\right)$ are defined. Due to the Poisson condition, the nilpotency of the exterior derivative, $d_{\theta}^{2}=0$, is guaranteed, so that the exterior derivative $d_{\theta}$ defines the $d_{\theta}$-cohomology. These operations satisfy the Cartan relations

$$
\left\{\bar{\iota}_{\xi}, \bar{\iota}_{\eta}\right\}=0, \quad\left\{d_{\theta}, \bar{\iota}_{\xi}\right\}=\overline{\mathcal{L}}_{\xi}, \quad\left[\overline{\mathcal{L}}_{\xi}, \bar{\iota}_{\eta}\right]=\bar{\iota}_{[\xi, \eta]_{\theta}}, \quad\left[\overline{\mathcal{L}}_{\xi}, \overline{\mathcal{L}}_{\eta}\right]=\overline{\mathcal{L}}_{[\xi, \eta]_{\theta}}, \quad\left[d_{\theta}, \overline{\mathcal{L}}_{\xi}\right]=0
$$

Contravariant Affine Connection Let us introduce a notion of contravariant affine connection $\bar{\nabla}$ on the Lie algebroid $\Gamma\left(T^{*} M\right)$. It is defined as a map satisfying

$$
\bar{\nabla}_{f \xi} \eta=f \bar{\nabla}_{\xi} \eta, \quad \bar{\nabla}_{\xi}(f \eta)=\left(\overline{\mathcal{L}}_{\xi} f\right) \eta+f \bar{\nabla}_{\xi} \eta,
$$

for any 1-forms $\xi, \eta$ and function $f$. Thus, the covariant derivatives are global objects and independent of our choice of local coordinates.

On some local patch $\left\{x^{i}\right\}$, the connection above is specified by the coefficients

$$
\bar{\nabla}_{d x^{i}} d x^{j}=\bar{\Gamma}_{k}^{i j} d x^{k},
$$

for one-form basis $\left\{d x^{i}\right\}$. On another local patch, say $\left\{x^{\prime i}\right\}$, we have another set of basis of one-forms $\left\{d x^{\prime i}\right\}$. For this basis, we introduce the coefficients $\bar{\Gamma}^{\prime}$ as

$$
\bar{\nabla}_{d x^{\prime a}} d x^{\prime b} \equiv\left(\bar{\Gamma}^{\prime}\right)_{c}^{a b} d x^{\prime c} .
$$

On the intersection of the two local patches $\left\{x^{i}\right\}$ and $\left\{x^{\prime i}\right\}$, on the other, we also have

$$
\bar{\nabla}_{d x^{\prime a}} d x^{\prime b}=\bar{\nabla}_{\frac{\partial x^{\prime a}}{\partial x^{i}} d x^{i}}\left(\frac{\partial x^{\prime b}}{\partial x^{j}} d x^{j}\right)=\frac{\partial x^{\prime a}}{\partial x^{i}}\left(\theta^{i j} \frac{\partial^{2} x^{\prime b}}{\partial x^{j} \partial x^{k}}+\frac{\partial x^{\prime b}}{\partial x^{j}} \bar{\Gamma}_{k}^{i j}\right) d x^{k},
$$

with a use of (A.3) and (A.4). Then we can extract the behavior of the coefficients under coordinate transformations. As usual, the coefficients do not behave as a tensor:

$$
\left(\bar{\Gamma}^{\prime}\right)_{c}^{a b}=\frac{\partial x^{k}}{\partial x^{\prime c}} \frac{\partial x^{\prime a}}{\partial x^{i}} \theta^{i j} \frac{\partial^{2} x^{\prime b}}{\partial x^{j} \partial x^{k}}+\frac{\partial x^{\prime a}}{\partial x^{i}} \frac{\partial x^{\prime b}}{\partial x^{j}} \frac{\partial x^{k}}{\partial x^{\prime c}} \bar{\Gamma}_{k}^{i j} .
$$

This property (A.7) implies that the subtraction $\bar{\Gamma}^{(1)}-\bar{\Gamma}^{(2)}$ of any two connections $\bar{\Gamma}^{(1)}$ and $\bar{\Gamma}^{(2)}$ behaves as a tensor of type $(2,1)$, as long as the same Poisson tensor is utilized. And also the trace of the connection coefficients $\left\{\bar{\Gamma}_{a}^{a b}\right\}$ defines a tensor of type $(1,0)$, i.e. a vector field. 
Torsion and Curvature The torsion of a contravariant affine connection $\bar{\nabla}$ is defined by

$$
\bar{T}(\xi, \eta)=\bar{\nabla}_{\xi} \eta-\bar{\nabla}_{\eta} \xi-[\xi, \eta]_{\theta}
$$

$\bar{T}$ turns out to be a tensor of type $(2,1)$, since it satisfies $\bar{T}(f \xi, \eta)=f \bar{T}(\xi, \eta)=\bar{T}(\xi, f \eta)$.

The curvature of a contravariant affine connection $\bar{\nabla}$ is defined by

$$
\bar{R}(\xi, \eta) \zeta=\left(\bar{\nabla}_{\xi} \bar{\nabla}_{\eta}-\bar{\nabla}_{\eta} \bar{\nabla}_{\xi}-\bar{\nabla}_{[\xi, \eta]_{\theta}}\right) \zeta
$$

It is easily shown that, for any functions $f, g$ and $h$, it satisfies $\bar{R}(f \xi, g \eta)(h \zeta)=f g h \bar{R}(\xi, \eta) \zeta$.

The curvature, together with the torsion tensor $\bar{T}$, satisfies the following Bianchi identities

$$
\begin{aligned}
& \mathfrak{S}\{\bar{R}(\xi, \eta) \zeta\}=\mathfrak{S}\left\{\left(\bar{\nabla}_{\zeta} \bar{T}\right)(\xi, \eta)+\bar{T}(\bar{T}(\xi, \eta), \zeta)\right\}, \\
& \mathfrak{S}\left\{\left(\bar{\nabla}_{\zeta} \bar{R}\right)(\xi, \eta)+\bar{R}(\bar{T}(\xi, \eta), \zeta)\right\}=0,
\end{aligned}
$$

where $\mathfrak{S}$ denotes the cyclic sum over $\xi, \eta$ and $\zeta$.

\section{B Relation between (Contravariant) Levi-Civita Connections}

The contravariant Levi-Civita connection is related to the usual Levi-Civita connection by

$$
\bar{\Gamma}_{k}^{i j}=\Gamma_{m k}^{j} \theta^{m i}+K_{k}^{i j}
$$

where $K_{k}^{i j}$ is understood as a contravariant version of contorsion tensor

$$
K_{k}^{i j}=G_{k l} K^{l i j}, \quad K^{k i j}=\frac{1}{2}\left(\nabla^{k} \theta^{i j}-\nabla^{i} \theta^{j k}+\nabla^{j} \theta^{k i}\right) .
$$

Here $\nabla$ denotes the ordinary usual Levi-Civita connection. The raising and lowering of the indices are done by the metric $G$ and $G^{-1}$ as usual, e.g. $\nabla^{i}=G^{i j} \nabla_{j}$. The contravariant Riemann tensor in terms of the usual Levi-Civita connection and the contorsion tensor reads

$$
\bar{R}_{l}^{k i j}=\theta^{i m} \theta^{n j} \mathrm{R}_{l m n}^{k}-\left(\nabla_{n} \theta^{i j}\right) K_{l}^{n k}+\theta^{n j} \nabla_{n} K_{l}^{i k}-\theta^{n i} \nabla_{n} K_{l}^{j k}+K_{m}^{j k} K_{l}^{i m}-K_{m}^{i k} K_{l}^{j m},
$$

where $\mathrm{R}_{l m n}^{k}$ is the ordinary Riemann tensor made out of the metric $G_{i j}$. By using the expression (B.3), the contravariant Ricci tensor and the scalar curvature are also written as

$$
\begin{aligned}
& \bar{R}^{k j}=\theta^{l m} \theta^{n j} \mathrm{R}_{l m n}^{k}-\left(\nabla_{n} \theta^{l j}\right) K_{l}^{n k}+\theta^{n j} \nabla_{n} \nabla_{l} \theta^{l k}-\theta^{n l} \nabla_{n} K_{l}^{j k}+K_{m}^{j k} \nabla_{l} \theta^{l m}-K_{m}^{l k} K_{l}^{j m}, \\
& \bar{R}=\theta^{l m} \theta^{n j} \mathrm{R}_{j l m n}+2 \theta_{n m} \nabla^{n} \nabla_{l} \theta^{l m}-\nabla^{n} \theta_{n m} \nabla_{l} \theta^{l m}
\end{aligned}
$$

where $\theta_{i j}=G_{i k} G_{j l} \theta^{k l}$.

\section{Computations on Two-dimensional Manifolds}

Some straightforward but lengthy computations are presented. 


\section{C.1 Calculation of Ricci Tensor}

Since the Riemann tensor $\bar{R}_{l}^{i j k}$ have the same structure as $R_{j k l}^{i}$, the only independent component of the Riemann tensor is

$$
\bar{R}^{1212}=\frac{1}{2}(\operatorname{det} G) \bar{R}
$$

i.e.

$$
\bar{R}^{i j k l}=\frac{1}{2}\left(G^{i k} G^{j l}-G^{i l} G^{j k}\right) \bar{R} .
$$

This equation implies that the vacuum Einstein equation (3.11) is automatically satisfied.

The Ricci scalar in the usual covariant language is given by (B.4), i.e.

$$
\bar{R}=\theta^{j k} \theta^{l i} R_{i j k l}+2 \theta_{k j} \nabla^{k} \nabla_{i} \theta^{i j}-\nabla^{k} \theta_{k j} \nabla_{i} \theta^{i j}
$$

where $R_{j l m n}$ is the Riemann tensor made out of $g_{i j}, \nabla$ denotes the usual Levi-Civita connection and the indices are lowered by the Riemannian metric $g_{i j}$ :

$$
\theta_{12}=G_{1 i} G_{2 j} \theta^{i j}=\left(G_{11} G_{22}-G_{12} G_{21}\right) \theta^{12}=\left(\operatorname{det} G^{-1}\right) \theta^{12}
$$

which implies

$$
\theta_{i j}=\epsilon_{i j}(\operatorname{det} g) \theta=\epsilon_{i j} \sqrt{g} \sigma
$$

Here we have introduced $\sigma=\sqrt{g} \theta$.

In the following we demonstrate explicit computations of each terms in (C.3). We can see immediately that the first term is

$$
\theta^{j k} \theta^{l i} R_{i j k l}=\theta^{2}(\operatorname{det} g) R
$$

To calculate the second and third terms, we consider a covariant derivative acting on the Poisson tensor:

$$
\begin{aligned}
\nabla_{k} \theta^{i j} & =\partial_{k} \theta^{i j}+\Gamma_{k l}^{i} \theta^{l j}+\Gamma_{k l}^{j} \theta^{i l} \\
& =\left(\partial_{k} \sigma\right) \frac{1}{\sqrt{g}} \epsilon^{i j}+\frac{1}{\sqrt{g}}\left(-\frac{1}{\sqrt{g}} \partial_{k} \sqrt{g} \epsilon^{i j}+\Gamma_{k m}^{i} \epsilon^{m j}+\Gamma_{k m}^{j} \epsilon^{i m}\right) .
\end{aligned}
$$

Since $\Gamma_{k l}^{i} \epsilon^{l j}+\Gamma_{k l}^{j} \epsilon^{i l}$ is antisymmetric under an exchange between $i$ and $j$, we have the relation

$$
\Gamma_{k l}^{i} \epsilon^{l j}+\Gamma_{k l}^{j} \epsilon^{i l}=\epsilon^{i j} \Gamma_{l k}^{l}=\epsilon^{i j} \frac{1}{\sqrt{g}} \partial_{k} \sqrt{g} .
$$

Therefore we find the covariant derivative on the Poisson tensor becomes

$$
\nabla_{k} \theta^{i j}=\left(\partial_{k} \sigma\right) \frac{1}{\sqrt{g}} \epsilon^{i j}
$$


Utilizing this, we have

$$
\nabla_{k} \theta_{i j}=G_{i m} G_{j n} \nabla_{k} \theta^{m n}=\epsilon_{i j} \sqrt{g} \partial_{k} \sigma
$$

Using above relations, we find the second and third terms in (C.3) become

$$
\begin{aligned}
& \theta_{i j} \nabla^{i} \nabla_{k} \theta^{k j}=\sigma \nabla^{i} \partial_{i} \sigma, \\
& \nabla^{i} \theta_{i j} \nabla_{k} \theta^{k j}=\partial^{i} \sigma \partial_{i} \sigma .
\end{aligned}
$$

Therefore, we get an explicit form of the Ricci scalar in terms of $\sigma$ :

$$
\bar{R}=\sigma^{2} R+2 \sigma \nabla^{i} \partial_{i} \sigma-\partial^{i} \sigma \partial_{i} \sigma
$$

\section{C.2 Calculation of Weyl Transformation}

In the following, we calculate $G_{k j} \bar{L}_{i}^{k i j}$ explicitly. On a two-dimensional manifold we find

$$
G_{k j} \bar{L}_{i}^{k i j}=G_{k j}\left(\delta_{i}^{j} B^{i k}-2 B^{j k}-G^{j k} G_{i m} B^{i m}+\delta_{m}^{j} B^{m k}\right)=-2 G_{i j} B^{i j},
$$

where

$$
\begin{aligned}
G_{i j} B^{i j} & =G_{i k}\left(\bar{\nabla}_{d x^{i}}\left(\theta^{k j} \partial_{j} \Omega\right)-\left(\theta^{i j} \partial_{j} \Omega\right)\left(\theta^{k l} \partial_{l} \Omega\right)+\frac{1}{2} G^{i k} G_{j l}\left(\theta^{j m} \partial_{m} \Omega\right)\left(\theta^{l n} \partial_{n} \Omega\right)\right) \\
& =\bar{\nabla}_{d x^{i}}\left(G_{i k} \theta^{k j} \partial_{j} \Omega\right) .
\end{aligned}
$$

Thus we see, with a use of (2.26),

$$
\begin{aligned}
\frac{1}{2} e^{\phi} \sqrt{g} G_{k j} \bar{L}_{i}^{k i j} & =-e^{\phi} \sqrt{g} \bar{\nabla} d x^{i}\left(G_{i k} \theta^{k j} \partial_{j} \Omega\right) \\
& =-\partial_{i}\left(e^{\phi} \sqrt{g} G_{i k} \theta^{k j} \partial_{j} \Omega\right) .
\end{aligned}
$$

Hence an additional term in (4.11) induced by a Weyl transform is just a surface term for any choice of $\Omega$. Furthermore, in the case (4.12) we have

$$
G_{i j} B^{i j}=\theta^{i l} \partial_{l}\left(\frac{G_{i k}}{\sigma} \theta^{k j} \partial_{j} \sigma\right)+\frac{1}{\sqrt{G^{-1}}} \partial_{i}\left(\sqrt{G^{-1}} \theta^{i l}\right) G_{l k} \theta^{k j} \frac{\partial_{j} \sigma}{\sigma} .
$$

Here we utilized the fact $\bar{\nabla}_{d x^{k}} G_{i j}=0$ and the formula (2.22). Substituting $\theta^{i j}=\frac{1}{\sqrt{G^{-1}}} \sigma \epsilon^{i j}$ and using $\epsilon^{i k} \epsilon^{j l} G_{k l}=G^{-1} G^{i j}$, we find that

$$
G_{k j} \bar{L}_{i}^{k i j}=2 G^{i j} \partial_{i} \sigma \partial_{j} \sigma-2 \sigma G^{i j} \nabla_{i} \partial_{j} \sigma
$$

and then

$$
\frac{1}{2} e^{\phi} \sqrt{g} G_{k j} \bar{L}_{i}^{k i j}=\sqrt{g} \frac{1}{\sigma^{2}}\left(G^{i j} \partial_{i} \sigma \partial_{j} \sigma-\sigma G^{i j} \nabla_{i} \partial_{j} \sigma\right) .
$$




\section{References}

[1] T. Asakawa, H. Muraki and S. Watamura, "Gravity theory on Poisson manifold with R-flux," Fortsch. Phys. 63 (2015) 683-704, arXiv:1508.05706 [hep-th].

[2] H. S. Snyder, "Quantized Space-time," Phys. Rev. 71 (1947) 38.

[3] A. P. Balachandran, T. R. Govindarajan, K. S. Gupta and S. Kurkcuoglu, "Noncommutative two dimensional gravities," Class. Quant. Grav.23 (2006) 5799-5810, arXiv:hep-th/0602265.

[4] E. Harikumar and V. O. Rivelles, "Noncommutative Gravity," Class. Quant. Grav.23 (2006) 7551-7560, arXiv: hep-th/0607115.

[5] M. R. Douglas and N. A. Nekrasov, "Noncommutative field theory," Rev. Mod. Phys. 73 (2001) 977-1029, arXiv:hep-th/0106048.

[6] R. Szabo, "Quantum field theory on noncommutative spaces," Phys. Rept. 378 (2003) 207-299, arXiv:hep-th/0109162.

[7] T. Yoneya, "On the interpretation of minimal length in string theories," Mod. Phys. Lett. A4 (1989) 1587.

[8] T. Yoneya, "Space-Time Uncertainty and Approaches to D-Brane Field Theory," Prog. Theor. Phys. Supp. 171 (2007) 87.

[9] S. Hossenfelder, "Minimal Length Scale Scenarios for Quantum Gravity," Living Rev. Rel. 16 (2013) 2, arXiv:1203.6191 [gr-qc].

[10] P. Aschieri, C. Blohmann, M. Dimitrijevic, F. Meyer, P. Schupp and J. Wess, "A Gravity theory on noncommutative spaces," Class. Quant. Grav. 22 (2005) 3511-3532, arXiv: hep-th/0504183.

[11] P. Aschieri, M. Dimitrijevic, F. Meyer and J. Wess, "Noncommutative geometry and gravity," Class. Quant. Grav. 23 (2006) 1883-1912, arXiv:hep-th/0510059.

[12] S. Kurkcuoglu and C. Saemann, "Drinfeld Twist and General Relativity with Fuzzy Spaces," Class. Quant. Grav.24 (2007) 291-312, arXiv:hep-th/0606197.

[13] T. Asakawa, H. Muraki, S. Sasa and S. Watamura, "Poisson-generalized geometry and R-flux," Int.J.Mod.Phys. A30 (2015) 17 1550097, arXiv:1408.2649 [hep-th].

[14] R. L. Fernandes, "Connections in Poisson geometry. I. Holonomy and invariants," J. Differential Geom. 542 (2000) 303-365, arXiv:math/0001129v2.

[15] M. Boucetta, "Poisson manifolds with compatible pseudo-metric and pseudo-Riemannian Lie algebras," Differential Geom. Appl. 203 (2004) 279-291, arXiv:math/0206102.

[16] M. Boucetta, "Riemann-Poisson manifolds and Kähler-Riemann foliations," C. R. Acad. Sci. Paris 3365 (2003) 423-428, arXiv:math/0211035.

[17] E. Hawkins, "Noncommutative rigidity," Comm. Math. Phys. 2462 (2004) 211-235, arXiv:math/0211203. 
[18] Y. Kosmann-Schwarzbach, "Poisson Manifolds, Lie Algebroids, Modular Classes: a Survey," SIGMA 4 (2008) 005, arXiv:0710.3098.

[19] M. Kontsevich, "Deformation quantization of Poisson manifolds. 1.," Lett. Math. Phys. 66 (2003) 157-216, arXiv:q-alg/9709040.

[20] N. Hitchin, "Generalized Calabi-Yau manifolds," Quart. J. Math. 54 (2003) 281-308, arXiv:math/0209099.

[21] A. Connes, "Gravity coupled with matter and foundation of noncommutative geometry," Commun. Math. Phys. 182 (1996) 155-176, arXiv:hep-th/9603053.

[22] P. Aschieri and L. Castellani, "Noncommutative Gravity Solutions," J. Geom. Phys. 60 (2010) 375-393, arXiv:0906.2774 [hep-th].

[23] T. Asakawa and S. Kobayashi, "Noncommutative Solitons of Gravity," Class. Quant. Grav. 27 (2010) 105014, arXiv:0911.2136 [hep-th].

[24] S. Kobayashi and T. Asakawa, "Angles in Fuzzy Disc and Angular Noncommutative Solitons," JHEP 04 (2013) 145, arXiv:1206.6602 [hep-th].

[25] H. S. Yang, "Emergent Gravity from Noncommutative Spacetime," Int. J. Mod. Phys. A24 (2009) 4473-4517, arXiv:hep-th/0611174.

[26] H. Steinacker, "Emergent Gravity from Noncommutative Gauge Theory," JHEP 12 (2007) 049, arXiv:0708.2426 [hep-th].

[27] H. Steinacker, "The curvature of branes, currents and gravity in matrix models," JHEP 01 (2013) 112, arXiv:1210.8364 [hep-th].

[28] L. Schneiderbauer and H. C. Steinacker, "Measuring finite Quantum Geometries via Quasi-Coherent States," J. Phys. A49 (2016) 28 285301, arXiv:1601.08007 [hep-th].

[29] G. Ishiki, "Matrix Geometry and Coherent States," Phys. Rev. D92 (2015) 4 046009, arXiv:1503.01230 [hep-th].

[30] G. Ishiki, T. Matsumoto and H. Muraki, "Kähler structure in the commutative limit of matrix geometry," JHEP 08 (2016) 042, arXiv:1603.09146 [hep-th].

[31] A. Iqbal, N. Nekrasov, A. Okounkov and C. Vafa, "Quantum foam and topological strings," JHEP 04 (2008) 011, arXiv:1603.09146 [hep-th].

[32] H. S. Yang, "Calabi-Yau manifolds from noncommutative Hermitian U(1) instantons," Phys. Rev. D91 (2015) 10, 104002, arXiv:1411.6115 [hep-th].

[33] H. S. Yang, "Mirror Symmetry in Emergent Gravity," arXiv:1412.1757 [hep-th].

[34] D. Andriot, M. Larfors, D. Lüst and P. Patalong, "A ten-dimensional action for non-geometric fluxes," JHEP 1109 (2011) 134, arXiv:1106.4015 [hep-th].

[35] D. Andriot, O. Hohm, M. Larfors, D. Lüst and P. Patalong, "A geometric action for non-geometric fluxes," Phys.Rev.Lett. 108 (2012) 261602, arXiv:1202.3060 [hep-th] . 
[36] D. Andriot, O. Hohm, M. Larfors, D. Lüst and P. Patalong, "Non-Geometric Fluxes in Supergravity and Double Field Theory," Fortsch.Phys. 60 (2012) 1150-1186, arXiv:1204.1979 [hep-th].

[37] R. Blumenhagen, A. Deser, E. Plauschinn and F. Rennecke, "Non-geometric strings, symplectic gravity and differential geometry of Lie algebroids," JHEP 1302 (2013) 122, arXiv:1211.0030 [hep-th].

[38] D. Andriot and A. Betz, " $\beta$-supergravity: a ten-dimensional theory with non-geometric fluxes, and its geometric framework," JHEP 1312 (2013) 083, arXiv:1306.4381 [hep-th].

[39] D. Andriot and A. Betz, "NS-branes, source corrected Bianchi identities, and more on backgrounds with non-geometric fluxes," JHEP 07 (2014) 059, arXiv:1402.5972 [hep-th].

[40] D. Andriot and A. Betz, "Supersymmetry with non-geometric fluxes, or a $\beta$-twist in Generalized Geometry and Dirac operator," JHEP 04 (2013) 006, arXiv:1411.6640 [hep-th].

[41] T. Bessho, M. A. Heller, N. Ikeda and S. Watamura, "Topological Membranes, Current Algebras and H-flux-R-flux Duality based on Courant Algebroids," JHEP 04 (2016) 170, arXiv: 1511.03425 [hep-th].

[42] A. S. Schwarz, "Geometry of Batalin-Vilkovisky quantization," Commun. Math. Phys. 155 (1993) 249-260, arXiv:hep-th/9205088.

[43] A. S. Schwarz, "Semiclassical approximation in Batalin-Vilkovisky formalism," Commun. Math. Phys. 158 (1993) 373-396, arXiv:hep-th/9210115.

[44] M. Alexandrov, M. Kontsevich, A. Schwartz and O. Zaboronsky, "The Geometry of the master equation and topological quantum field theory," Int. J. Mod. Phys. A12 (1997) 1405-1430, arXiv:hep-th/9502010.

[45] N. Ikeda, "Lectures on AKSZ Sigma Models for Physicists," arXiv:1204.3714 [hep-th].

[46] U. Carow-Watamura, M. A. Heller, N. Ikeda, Y. Kaneko and S. Watamura, "Higher Gauge Theories from Lie n-algebras and Off-Shell Covariantization," JHEP 07 (2016) 125, arXiv:1606.03861 [hep-th].

[47] T. Asakawa, H. Muraki and S. Watamura, "Topological T-duality via Lie algebroids and Q-flux in Poisson-generalized geometry," Int.J.Mod.Phys. A30 (2015) 30 1550182, arXiv: 1503.05720 [hep-th].

[48] B. Jurčo and J. Vysoký, "Leibniz algebroids, generalized Bismut connections and Einstein-Hilbert actions," J. Geom. Phys. 97 (2015) 25-33, arXiv:1503.03069 [hep-th].

[49] B. Jurčo and J. Vysoký, "Heterotic reduction of Courant algebroid connections and Einstein-Hilbert actions," Nucl. Phys. B909 (2016) 86-121, arXiv:1512.08522 [hep-th]. 